\title{
Parity-violating electron scattering at the MAMI facility in Mainz
}

\section{The strangeness contribution to the form factors of the nucleon}

\author{
F.E. Maas ${ }^{\mathrm{a}}$
}

For the A4-Collaboration

Institut de Physique Nucléaire, CNRS/IN2P3, Université de Paris Sud, 15, rue Georges Clemenceau, F-91406 Orsay CEDEX, France

\author{
/ \\ Published online: 29 May 2006 - (c) Società Italiana di Fisica / Springer-Verlag 2006
}

\begin{abstract}
A measurement of the weak form factor of the proton allows a separation of the strangeness contribution to the electromagnetic form factors. The weak form factor is accessed experimentally by the measurement of a parity violating (PV) asymmetry in the scattering of polarized electrons on unpolarized protons. We performed such measurements with the setup of the A4-experiment at the MAMI accelerator facility in Mainz. The role of strangeness in low energy nonperturbative QCD is discussed. The A4experiment is presented as well as the results on the strangeness form factors which have been measured at two $Q^{2}$-values. The plans for backward angle measurements at the MAMI facility are presented.
\end{abstract}

PACS. 12.15.-y Electroweak interactions - 11.30.Er Charge conjugation, parity, time reversal, and other discrete symmetries - 13.40.Gp Electromagnetic form factors - 25.30.Bf Elastic electron scattering

\section{Introduction}

In 1965 , the discovery of weak interaction violating the symmetry transformation of parity $P: \boldsymbol{x} \rightarrow-\boldsymbol{x}[1,2]$ has opened a new door in studying the weak force. In contrast electromagnetic and strong interaction conserve parity and therefore parity non-conserving observables are an important tool to uniquely identify weak interaction. Parityviolating correlation observables like pseudo-scalars allow one in electron scattering to access the weak neutral current and separate it from the electromagnetic current and the associated photon exchange. Such an observable is for example the cross section asymmetry in scattering righthanded versus left-handed electrons off an unpolarized target. The first pioneering parity-violation (PV) experiment in electron scattering at high energy at SLAC [3], and at low energies at the MIT Bates accelerator [4] and at the Mainz linac [5] were intended to study the standard model. These experiments were ground breaking in developing techniques for measuring the small (order $10^{-4}$ ) asymmetries with order $10^{-5}$ errors.

Today, parity-violating observables are studied in many areas of physics, from atomic physics to nuclear physics, from hadron physics to high-energy physics [6]. The availability of a polarized high-intensity continuous electron beam at the Mainz Microtron MAMI was important to increase the sensitivity in measuring a parityviolating asymmetry by two orders of magnitude to a level of $0.1 \mathrm{ppm}$. The standard model and its parameters are

a e-mail: maas@ipno.in2p3.fr tested to an extent that the weak neutral current of the nucleon can be used to study nucleon structure. An experimental program to measure the strangeness contribution to the form factors at TJLab and at the MAMI facility has emerged. In this contribution to the proceedings we will focus on the experimental activity of the Mainz program and the results achieved with the A4 experiment. The parity program at MIT-Bates and at TJLab will be reviewed in another contribution to these proceedings (see the contribution of S. Kowalski).

\section{Strangeness in the nucleon}

Quantum chromodynamics (QCD) is the quantum field description of strong interaction. Quarks carrying color charge interact via the emission of gluons. The gluon is like the photon a spin-1 particle and it couples like the photon to the vector current of quarks. Similar to Quantum Electrodynamics (QED) an expansion of the interaction in a power series of the coupling constant $\alpha_{s}=\alpha_{\mathrm{QCD}}$ is possible. The renormalization of the Lagrangian leads in the case of QED to a running coupling $\alpha_{\mathrm{QED}}\left(Q^{2}\right)$, which rises slowly from the low-energy value of $\alpha_{\mathrm{QED}} \approx 1 / 137$ to the Z-mass where it reaches $\alpha_{\mathrm{QED}}\left(Q^{2} \approx m_{Z}^{2}\right) \approx 1 / 128$ [7]. The gluons carry color charge and this coupling leads to a very much different running of the coupling $\alpha_{\mathrm{QCD}}\left(Q^{2}\right)$, which is depicted in fig. 1. For scales $Q^{2}>1(\mathrm{GeV} / c)^{2}$, $\alpha_{\mathrm{QCD}}\left(Q^{2}\right)$ becomes small and perturbative treatment of QCD ("perturbative QCD") is possible [8] with the well 


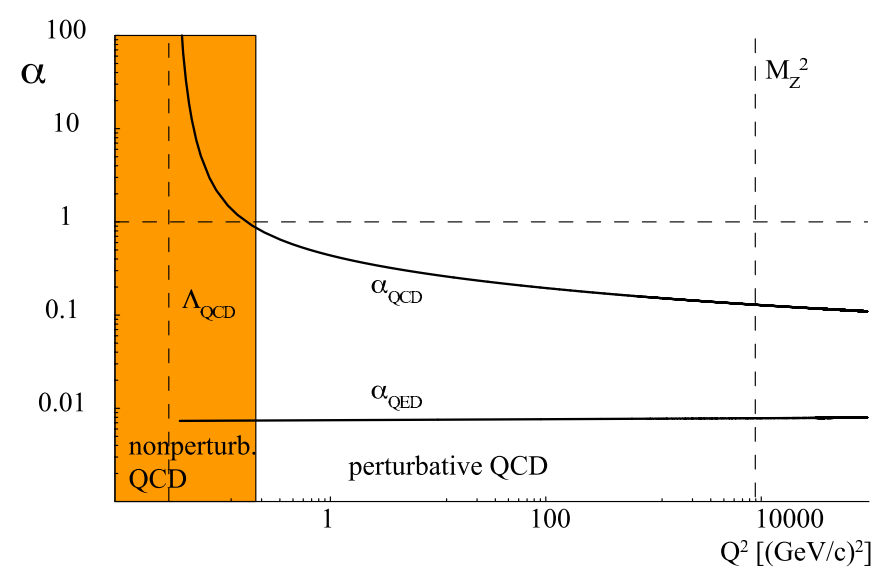

Fig. 1. Running coupling in QED $\left(\alpha_{\mathrm{QED}}\right)$ and QCD $\left(\alpha_{\mathrm{QCD}}\right)$ up to one loop. World data suggest that $\Lambda_{\mathrm{QCD}} \approx 250 \mathrm{MeV}$ analyzed in the $\overline{M S}$-renormalization scheme using 5 quark flavors [7].

known "asymptotic freedom" at higher scales [9]. An associated energy scale appears at low energies, $\Lambda_{\mathrm{QCD}}$, at which $\alpha_{\mathrm{QCD}}\left(Q^{2}\right)$ diverges. Any perturbative description breaks down, the theory is here not solvable, this is the regime of nonperturbative QCD. Virtual excitations of pairs of quarks and antiquarks play an important role in the range of nonperturbative QCD. Understanding QCD in this regime is closely linked to the structure of the matter surrounding us like proton and neutron. The successful description of a wide variety of observables by the concept of effective, heavy ( $\approx 350 \mathrm{MeV}$ ) constituent quarks, which are not the current quarks of QCD, is still a puzzle. There are other equivalent descriptions of hadronic matter at low energy scales in terms of effective fields like chiral perturbation theory $(\chi \mathrm{PT})$ or Skyrme-type soliton models. The effective fields in these models arise dynamically from a sea of virtual gluons and quark-antiquark pairs.

In this context the contribution of strange quarks plays a special role since the nucleon has no net strangeness, and any contribution of strange quarks to nucleon structure observables is a pure sea-quark effect and can not be clouded by valence quark effects. Due to the heavier current quark mass of strangeness $\left(m_{s}\right)$ as compared to up $\left(m_{u}\right)$ and down $\left(m_{d}\right)$ with $m_{s} \approx 140 \mathrm{MeV} \gg m_{u}, m_{d} \approx$ $5-10 \mathrm{MeV}$, one expects a suppression of strangeness effects in the creation of quark-antiquark pairs. On the other hand, the strange quark mass is in the range of the mass scale of QCD $\left(m_{s} \approx \Lambda_{\mathrm{QCD}}\right)$ so that the dynamic creation of strange sea quark pairs could still be substantial. The consequence of the presence of virtual strangeness quark pairs on different matrix elements has been studied:

For example, the strange density of the vacuum $\langle\bar{s} s\rangle$ is around $20 \%$ suppressed as compared to $\mathrm{u}$ - and d-quark condensates: $\langle\bar{s} s\rangle /\langle\bar{q} q\rangle=0.8 \pm 0.3$ [10], which supports the mechanism indicated above. The strange scalar density in the nucleon $\langle\bar{N}|\bar{s} s| N\rangle$ is studied in connection with the pion nucleon sigma term. The scalar strangeness content of the nucleon is defined as $y=2\langle\bar{p}|\bar{s} s| p\rangle /\langle p|\bar{u} u+\bar{d} d| p\rangle$, and a recent evaluation of $\pi \mathrm{N}$ scattering length data yields a value of $y=0.46$ corresponding to a contribution of the strange scalar density to the nucleon mass of $220 \mathrm{MeV}$ [11]. This contribution from scalar density to the nucleon mass is most likely cancelled by other contributions like potential energy and kinetic energy to an extend that the net strangeness contribution to the nucleon mass might be small [12].

The strangeness contribution to unpolarized nucleon structure functions has been determined in deep inelastic neutrino scattering. One obtains the momentum fraction of strange quarks as compared to $\mathrm{u}$ - and d-quarks: $\kappa=\langle x(s(x)+\bar{s}(x))\rangle /\langle x(\bar{u}(x)+\bar{d}(x))\rangle \approx 0.5[13,14]$. This corresponds to the fact, that at a scale of $Q^{2}=5(\mathrm{GeV} / c)^{2}$ the strange sea carries about $3 \%$ of the nucleons momentum. In a dynamical QCD evolution model one starts with some unpolarized quark structure function at a scale of $\mu=0.3(\mathrm{GeV} / c)^{2}$ and evolutes it to the scale of $Q^{2}=5(\mathrm{GeV} / c)^{2}$ where finally the measured parton distributions have been reproduced [15]. In this approach one finds, that the observed value of $\kappa \approx 0.5$ is compatible with a vanishing strange sea contribution at the low scale $\mu$.

Information on the axial charge $\left\langle N\left|\bar{s} \gamma_{\mu} \gamma^{5} s\right| N\right\rangle$ and on the strangeness contribution to the spin of the nucleon comes from the interpretation of deep inelastic scattering data and suggests a sizeable contribution of the strange quarks of $\Delta s\left(Q^{2}=1(\mathrm{GeV} / c)^{2}\right)=-4.5 \pm 0.7$ to the nucleon spin from a next-to-leading order perturbative QCD analysis of the available world data set including higher twist effects [16].

\section{Strangeness contribution to the form factors of the nucleon}

Parity-violating (PV) electron scattering off nucleons provides experimental access to the strange quark vector current in the nucleon $\left\langle N\left|\bar{s} \gamma_{\mu} s\right| N\right\rangle$ which is parameterized in the electromagnetic form factors of proton and neutron, $G_{E}^{s}$ and $G_{M}^{s}$ [17]. Recently the SAMPLE-, HAPPEX-, A4- and G0-collaborations have published experimental results. A direct separation of electric $\left(G_{E}^{s}\right)$ and magnetic $\left(G_{M}^{s}\right)$ contribution at forward angle has been impossible so far, since the measurements have been taken at different $Q^{2}$-values. The experimental details of the SAMPLE, HAPPEX and G0 collaboration are discussed in a different contribution to these proceedings (see the contribution of S. Kowalski).

A determination of the weak vector form factors of the proton $\left(\tilde{G}_{E}^{p}\right.$ and $\left.\tilde{G}_{M}^{p}\right)$ is done by measuring the PV asymmetry in the scattering of longitudinally polarized electron off unpolarized protons. It allows the determination of the strangeness contribution to the electromagnetic form factors $G_{E}^{s}$ and $G_{M}^{s}$. The weak vector form factors $\tilde{G}_{E, M}^{p}$ of the proton can be expressed in terms of the known electromagnetic nucleon form factors $G_{E, M}^{p, n}$ and the unknown strange form factors $G_{E, M}^{s}$ using isospin symmetry and the universality of the quarks in weak and electromagnetic interaction $\tilde{G}_{E, M}^{p}=\left(G_{E, M}^{p}-G_{E, M}^{n}\right)-$ $4 \sin ^{2} \theta_{W} G_{E, M}^{p}-G_{E, M}^{s}$. The interference between weak 
$(Z)$ and electromagnetic $(\gamma)$ amplitudes leads to a PV asymmetry $A_{L R}(\boldsymbol{e} p)=\left(\sigma_{R}-\sigma_{L}\right) /\left(\sigma_{R}+\sigma_{L}\right)$ in the elastic scattering cross section of right- and left-handed electrons ( $\sigma_{R}$ and $\sigma_{L}$ respectively), which is given in the framework of the Standard Model [18]. $A_{L R}(\boldsymbol{e p})$ is of order parts per million (ppm). The asymmetry can be expressed as a sum of three terms, $A_{L R}(e p)=A_{V}+A_{s}+A_{A}$.

$$
\begin{array}{r}
A_{V}=-a \rho_{e q}^{\prime}\left\{\left(1-4 \hat{\kappa}_{e q}^{\prime} \hat{s}_{Z}^{2}\right)-\frac{\epsilon G_{E}^{p} G_{E}^{n}+\tau G_{M}^{p} G_{M}^{n}}{\epsilon\left(G_{E}^{p}\right)^{2}+\tau\left(G_{M}^{p}\right)^{2}}\right\} \\
A_{s}=a \rho_{e q}^{\prime} \frac{\epsilon G_{E}^{p} G_{E}^{s}+\tau G_{M}^{p} G_{M}^{s}}{\epsilon\left(G_{E}^{p}\right)^{2}+\tau\left(G_{M}^{p}\right)^{2}} \\
A_{A}=a \frac{\left(1-4 \hat{s}_{Z}^{2}\right) \sqrt{1-\epsilon^{2}} \sqrt{\tau(1+\tau)} G_{M}^{p} \tilde{G}_{A}^{p}}{\epsilon\left(G_{E}^{p}\right)^{2}+\tau\left(G_{M}^{p}\right)^{2}} .
\end{array}
$$

$A_{V}$ arises from the $\mathrm{Z}_{0}$ coupling to the proton vector current and contains the electromagnetic nucleon form factors $G_{E, M}^{p, n}$. A possible strangeness contribution to the proton electromagnetic vector form factors has been separated into $A_{s}$. The coupling to the proton axial current is presented by $A_{A}$ and contains the neutral current weak axial form factor $\tilde{G}_{A}^{p}$. $\theta_{e}$ is the scattering angle of the electron in the laboratory and $Q^{2}$ the negative square of the four momentum transfer. $\tau=Q^{2} /\left(4 M_{p}^{2}\right)$ and $\epsilon=\left[1+2(1+\tau) \tan ^{2}\left(\theta_{e} / 2\right)\right]^{-1}$ represent purely kinematical factors with $M_{p}$ the proton mass. $G_{\mu}$ and $\alpha$ represent the Fermi coupling constant as derived from muon decay and the fine structure constant respectively. $a$ denotes the factor $\left(G_{\mu} Q^{2}\right) /(4 \pi \alpha \sqrt{2})$. In order to average $A_{0}=A_{V}+A_{A}$ over the acceptance of the detector and the target length, we take values for the electromagnetic form factors $G_{E, M}^{p, n}$ from a parametrization (version 1, page 5) by Friedrich and Walcher [19] and assign an experimental error of $3 \%$ to $G_{M}^{p}$ and $G_{E}^{p}, 5 \%$ to $G_{M}^{n}$, and $10 \%$ to $G_{E}^{n}$. For evaluating $A_{0}$, electromagnetic internal and external radiative corrections to the asymmetry and energy loss due to ionization in the target have been calculated and they reduce the expected asymmetry for our kinematics by $1.3 \%$.

Electro-weak quantum corrections have been applied in the $\overline{M S}$ renormalization scheme according to [20] and are contained in the factors $\rho_{\text {eq }}^{\prime}$, with $\hat{s}_{Z}^{2}=$ $\sin ^{2} \hat{\theta}_{W}\left(M_{Z}\right)_{\overline{M S}}=0.23120(15)$ [21]. The electro-weak quantum corrections to $A_{A}[22]$ are applied and absorbed in the value of $\tilde{G}_{A}^{p}$.

The largest contribution to the uncertainty of $A_{0}$ comes from the uncertainty in the axial form factor $\tilde{G}_{A}^{p}$, the electric form factor of the proton $G_{E}^{p}$, and the magnetic form factor of the neutron $G_{M}^{n}$. For our experimental program at backward angles, the uncertainty stemming from $\tilde{G}_{A}^{p}$ will be more important in the separation of $G_{E}^{s}$ and $G_{M}^{s}$. Using a different target like deuterium will allow to separate $\tilde{G}_{A}^{p}, G_{E}^{s}$ and $G_{M}^{s}$. The parity admixture in the ground state of deuterium is negligible [23].

\section{The A4 experimental setup and analysis}

The A4 experiment at MAMI has been developed and build in order to measure a small (order ppm) parity-

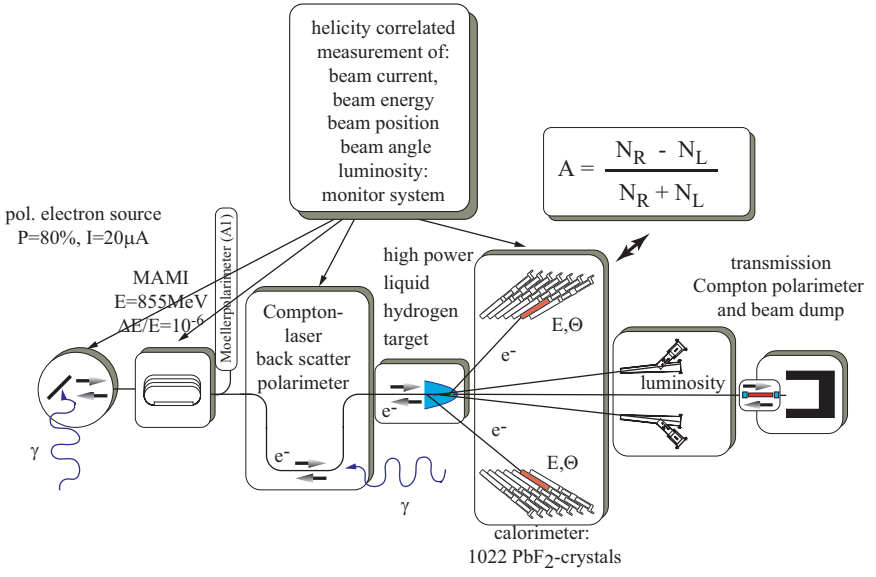

Fig. 2. Measurement principle of the A4 experiment. The polarized electrons from the source are accelerated in the MAMI accelerator to a maximum energy of $855 \mathrm{MeV}$. Scattered electrons from the $10 \mathrm{~cm}$ hydrogen target are detected in the homogeneous 1022 channel $\mathrm{PbF}_{2}$-Cherenkov calorimeter. The sensitive measurement and stabilization of all electron beam parameters is crucial for the sensitivity of the experiment.

violating cross section asymmetry in the scattering of polarized electrons off unpolarized protons. It is complementary to other experiments since for the first time counting techniques have been used in a PV electron scattering experiment. Possible systematic contributions to the experimental asymmetries and the associated uncertainties are of a different nature as compared to previous experiments, which use analogue integrating techniques. Figure 2 shows the measurement principle of the A4 experiment.

The polarized 570.4 and $854.3 \mathrm{MeV}$ electrons were produced using a strained layer GaAs crystal that is illuminated with circularly polarized laser light [24]. Average beam polarization was about $80 \%$. The helicity of the electron beam was selected every $20.08 \mathrm{~ms}$ by setting the high voltage of a fast Pockels cell according to a randomly selected pattern of four helicity states, either $(+P-P-P+P)$ or $(-P+P+P-P)$. A 20 ms time window enabled the histogramming in all detector channels and an integration circuit in the beam monitoring and luminosity monitoring systems. The exact window length was locked to the power frequency of $50 \mathrm{~Hz}$ in the laboratory by a phase locked loop. For normalization, the gate length was measured for each helicity. Between each $20 \mathrm{~ms}$ measurement gate, there was an $80 \mu$ s time window for the high voltage at the Pockels cell to be changed. The intensity $I=20 \mu \mathrm{A}$ of the electron current was stabilized to better than $\delta I / I \approx 10^{-3}$. An additional $\lambda / 2$-plate in the optical system was used to rotate small remaining linear polarization components and to control the helicity correlated asymmetry in the electron beam current to the level of $<10 \mathrm{ppm}$ in each five minute run.

From the source to the target, the electron beam develops fluctuations in beam parameters such as position, energy and intensity which are partly correlated to the reversal of the helicity from $+P$ to $-P$. We have used a system of microwave resonators in order to monitor beam 


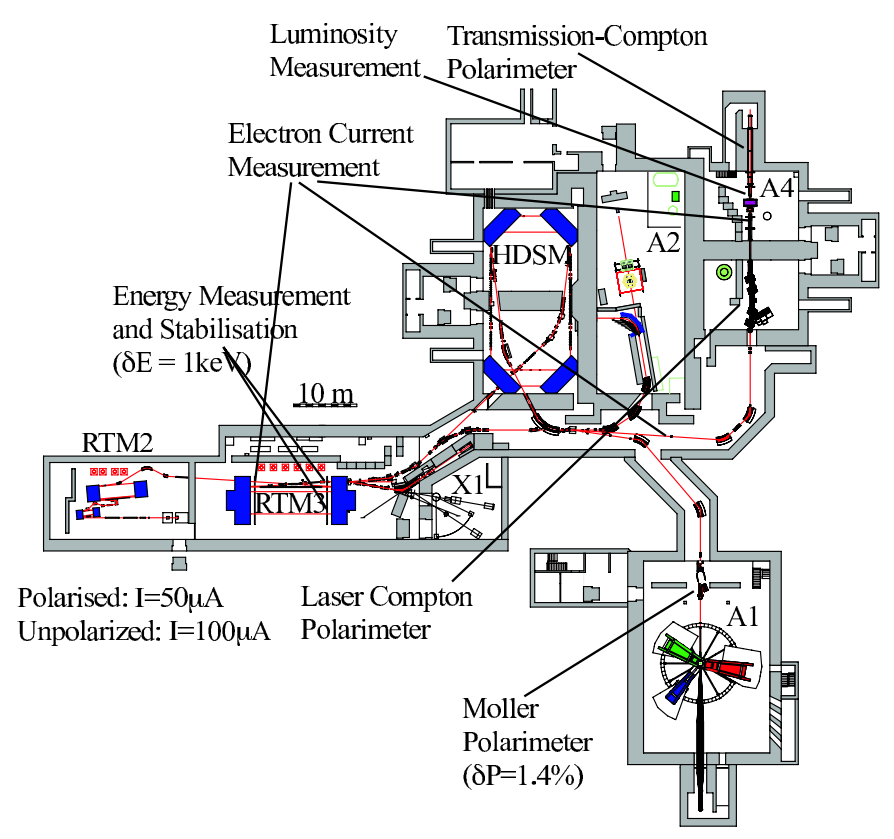

Fig. 3. Floor plan of the MAMI accelerator with the three race track microtrons (RTM). The A4 experiment is located in experimental halls 3 and 4 . Feedback stabilization systems for energy, position, angle and current had been developed for the A4 experiment.

current, energy, and position in two sets of monitors separated by a drift space of about $7.21 \mathrm{~m}$ in front of the hydrogen target. In addition, we have used a system of 10 feed-back loops in order to stabilize current, energy [25], position, and angle of the beam. Figure 3 shows an outline of the MAMI floor plan with the location of the A4 experiment and the beam monitoring and stabilisation systems.

The polarization of the electron beam was measured with an accuracy of $2 \%$ using a Møller polarimeter which is located on a beam line in another experimental hall [26]. Due to the fact that we had to interpolate between the weekly Møller measurements, the uncertainty in the knowledge of the beam polarization increased to $4 \%$.

The $10 \mathrm{~cm}$ high-power, high-flow liquid-hydrogen target was optimized to guarantee a high degree of turbulence with a Reynolds-number of $R>2 \times 10^{5}$ in the target cell in order to increase the effective heat transfer. For the first time, a fast modulation of the beam position of the intense CW $20 \mu \mathrm{A}$ beam could be avoided. It allowed us to stabilize the beam position on the target cell without target density fluctuations arising from boiling. The total thickness of the entrance and exit aluminum windows was $250 \mu \mathrm{m}$. The luminosity $L$ was monitored for each helicity state $(\mathrm{R}, \mathrm{L})$ during the experiment using eight waterCherenkov detectors (LuMo) that detect scattered particles symmetrically around the electron beam for small scattering angles in the range of $\theta_{e}=4.4^{\circ}-10^{\circ}$, where the PV asymmetry is negligible. The photomultiplier tube currents of these luminosity detectors were integrated during the $20 \mathrm{~ms}$ measurement period by gated integrators and then digitized by customized 16-bit analogue-todigital converters (ADC). The same method was used for

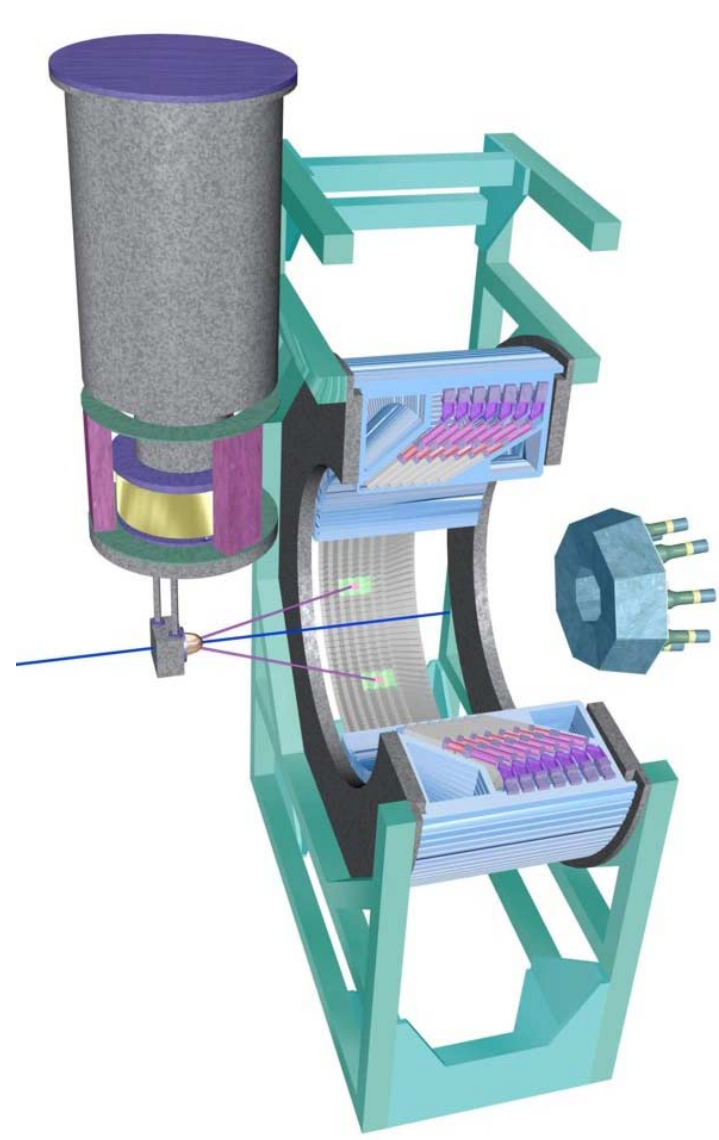

Fig. 4. The electron beam enters from the left and hits the hydrogen target at about $2.2 \mathrm{~m}$ above ground. Scattered electrons are detected with the 1022 channel $\mathrm{PbF}_{2}$-calorimeter, which covers a scattering angle of $30^{\circ}<\theta_{e}<40^{\circ}$ and an azimuthal angle of $360^{\circ}$. Part of the detector has been left out in the drawing for better visibility.

all the beam parameter signals. A correction was applied for the nonlinearity of the luminosity monitor photomultiplier tubes. From the beam current helicity pair data $I^{R, L}$ and luminosity monitor helicity pair $L^{R, L}$ data we calculated the target density $\rho^{R, L}=L^{R, L} / I^{R, L}$ for the two helicity states independently.

To detect the scattered electrons we developed a new type of a very fast, homogeneous, total absorption calorimeter consisting of individual lead fluoride $\left(\mathrm{PbF}_{2}\right)$ crystals $[27,28]$. Figure 4 shows a CAD-drawing of the calorimeter together with the hydrogen target. The material is a pure Cherenkov radiator and has been chosen for its fast timing characteristics and its radiation hardness. This is the first time this material has been used in a large scale calorimeter for a physics experiment. The crystals are dimensioned so that an electron deposits $96 \%$ of its total energy in an electromagnetic shower extending over a matrix of $3 \times 3$ crystals. Together with the readout electronics this allows us a measurement of the particle energy with a resolution of $3.9 \% / \sqrt{E}$ and a total dead time of $20 \mathrm{~ns}$. For the data taken at $854.3 \mathrm{MeV}$ only 511 out of 1022 channels of the detector and the readout electronics were operational, for the $570.4 \mathrm{MeV}$ data all 


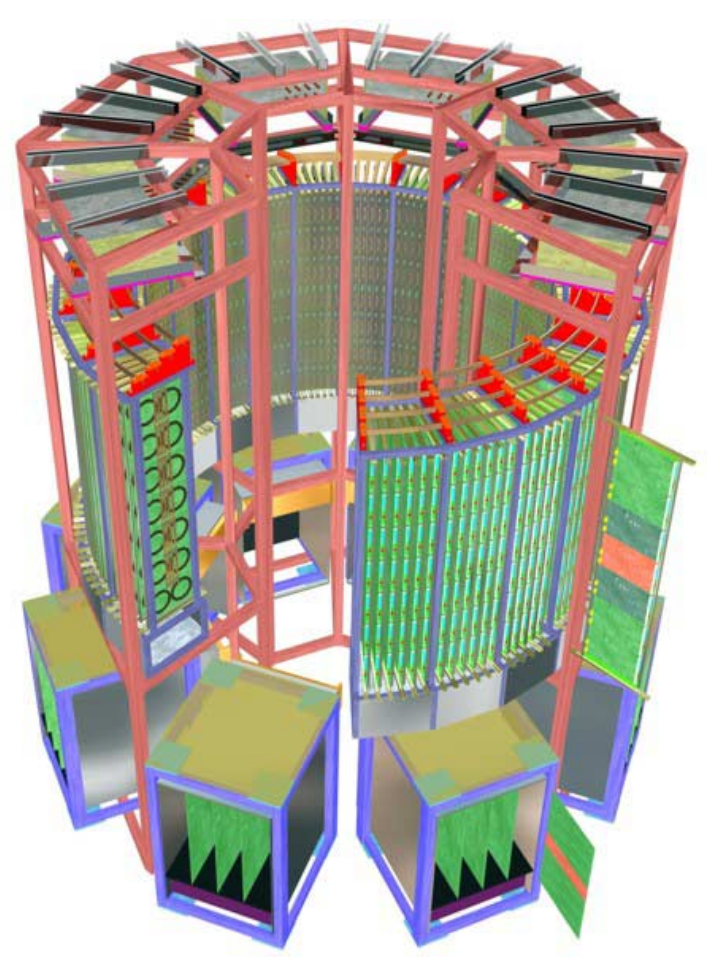

Fig. 5. Design drawing of the A4 readout electronics. The upper part contains the analog sum, trigger and veto circuits together with the digitization. In the lower part, the histogramming and the VMEbus access is done. The system is about $3.5 \mathrm{~m}$ high.

the 1022 channels were installed. The particle rate within the acceptance of this solid angle was $\approx 50 \times 10^{6} \mathrm{~s}^{-1}$. Due to the short dead time, the losses due to double hits in the calorimeter were $1 \%$ at $20 \mu \mathrm{A}$. This low dead time is only possible because of the special readout electronics employed. The signals from each cluster of 9 crystals were summed and integrated for $20 \mathrm{~ns}$ in an analogue summing and triggering circuit and digitized by a transient 8-bit ADC. There was one summation, triggering, and digitization circuit per crystal. The energy, helicity, and impact information were stored together in a three dimensional histogram. Neighboring crystals have to go to neighboring electronics channels in the electronics resulting a ring shape. Analogue summation and digitization has been galvanically separated from histogramming and VMEbus access. Figure 5 shows a design drawing of the fast A4 experiment electronics.

Figure 6 shows an energy spectrum of scattered particles. The number of elastic scattered electrons is determined from this histogram for each detector channel by integrating the number of events in an interval from $1.6 \sigma_{E}$ above pion production threshold to $2.0 \sigma_{E}$ above the elastic peak in each helicity histogram, where $\sigma_{E}$ is the energy resolution for nine crystals. These cuts ensure a clean separation between elastic scattering and pion production or $\Delta$-excitation which has an unknown PV cross section asymmetry. The linearity of the $\mathrm{PbF}_{2}$ detector system with respect to particle counting rates and possible effects

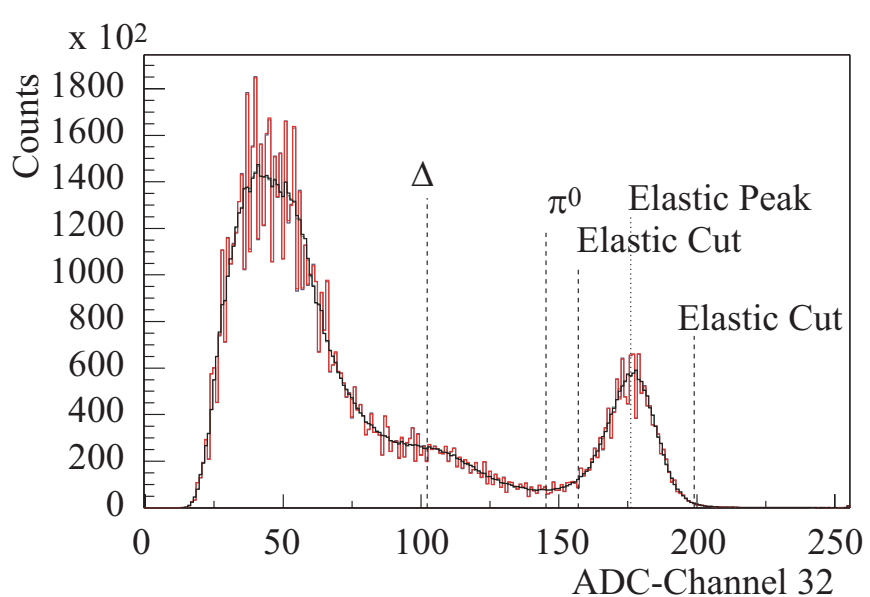

Fig. 6. The dashed histogram shows a raw energy spectrum of accepted particles from the hydrogen target as read directly from the hardware memory of the readout electronics of the lead fluoride calorimeter. For the solid black curve, the raw spectrum has been corrected for the differential nonlinearity of the ADC, i.e. for measured variations of the ADC channel width. The elastic scattering peak position, the $\pi^{0}$-production threshold and the $\Delta$-resonance position are indicated as well as the lower and upper cut position for the extraction of $N_{e}^{R}$ and $N_{e}^{L}$ as described in the text.

due to dead time were investigated by varying the beam current. We calculate the raw normalized detector asymmetry as $A_{\text {raw }}=\left(N_{e}^{R} / \rho^{R}-N_{e}^{L} / \rho^{L}\right) /\left(N_{e}^{R} / \rho^{R}+N_{e}^{L} / \rho^{L}\right)$. The possible dilution of the measured asymmetry by background originating from the production of $\pi^{0}$ s that subsequently decays into two photons where one of the photons carries almost the full energy of an elastic scattered electron was estimated using Monte Carlo simulations to be much less than $1 \%$ and is neglected here. The largest background comes from quasi-elastic scattering at the thin aluminum entrance and exit windows of the target cell. We have measured the aluminum quasi-elastic event rate and calculated in a static approximation a correction factor for the aluminum of $1.030 \pm 0.003$ giving a smaller value for the corrected asymmetry.

Corrections due to false asymmetries arising from helicity correlated changes of beam parameters were applied on a run by run basis. The analysis was based on the five minute runs for which the counted elastic events in the $\mathrm{PbF}_{2}$ detector were combined with the correlated beam parameter and luminosity measurements. In the analysis we applied reasonable cuts in order to exclude runs where the accelerator or parts of the $\mathrm{PbF}_{2}$ detector system were malfunctioning. The analysis is based on a total of $7.3 \times 10^{6}$ histograms corresponding to $4.8 \times 10^{12}$ elastic scattering events for the $854.3 \mathrm{MeV}$ data and $4.8 \cdot 10^{6}$ histograms corresponding to $2 \cdot 10^{13}$ elastic events for the $570.4 \mathrm{MeV}$ data.

For the correction of helicity correlated beam parameter fluctuations we used multi-dimensional linear regression analysis using the data. The regression parameters have been calculated in addition from the geometry of the 

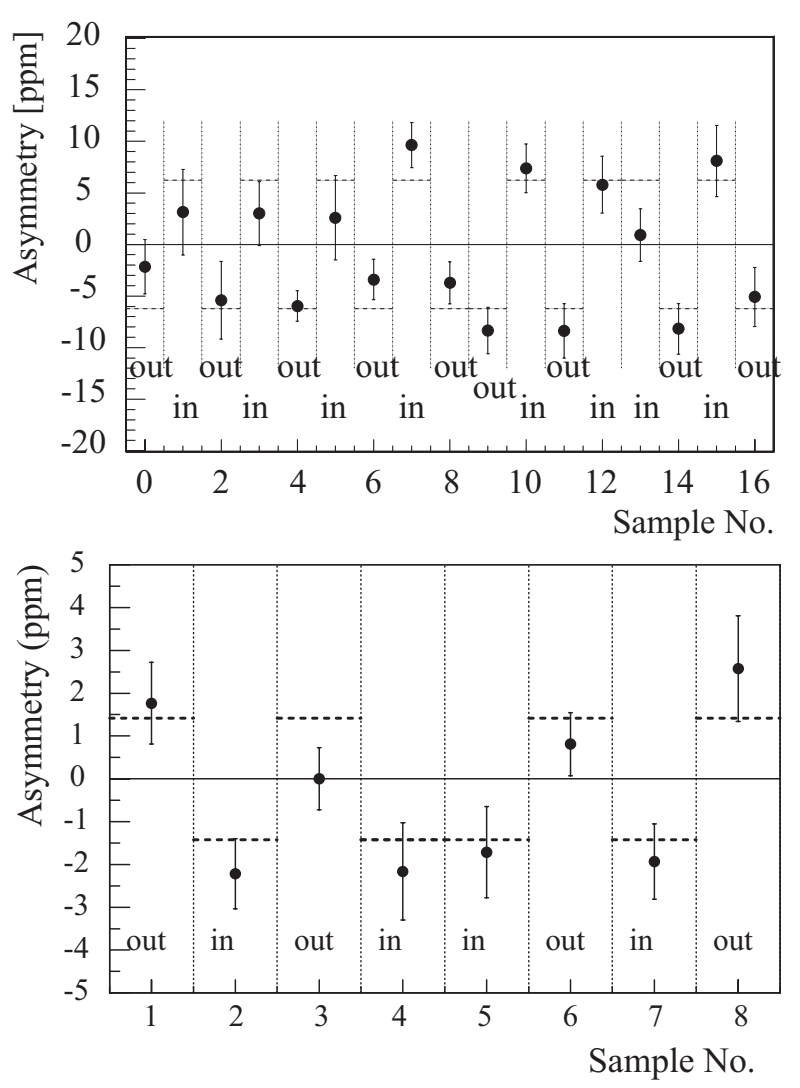

Fig. 7. The top plot shows the data samples of $854.3 \mathrm{MeV}$ data with the $\lambda / 2$-plate in and out. The lower plots represents the data sample for the $570.4 \mathrm{MeV}$ data with the $\lambda / 2$-plate in and out as described in the text.

precisely surveyed detector geometry. The two different methods agree very well within statistics.

The experimental asymmetry is normalized to the electron beam polarization $P_{e}$ to extract the physics asymmetry, $A_{\text {phys }}=A_{\exp } / P_{e}$. We have taken half of our data with a second $\lambda / 2$-plate inserted between the laser system and the GaAs crystal. This reverses the polarization of the electron beam and allows a stringent test of the understanding of systematic effects. The effect of the plate can be seen in fig. 7: the observed asymmetry extracted from the different data samples changes sign, which is a clear sign of parity violation if, as in our case, the target is unpolarized.

Our measured result for the PV physics asymmetry in the scattering cross section of polarized electrons on unpolarized protons at an average $Q^{2}$ value of $0.230(\mathrm{GeV} / c)^{2}$ is $A_{L R}(e p)=\left(-5.44 \pm 0.54_{\text {stat }} \pm 0.26_{\text {syst }}\right) \mathrm{ppm}$ for the $854.3 \mathrm{MeV}$ data [29] and $A_{L R}(\boldsymbol{e p})=\left(-1.36 \pm 0.29_{\text {stat }} \pm\right.$ $\left.0.13_{\text {syst }}\right) \mathrm{ppm}$ for the $570.4 \mathrm{MeV}$ data [30]. The first error represents the statistical accuracy, and the second represents the systematical uncertainties including beam polarization. The absolute accuracy of the experiment represents the most accurate measurement of a PV asymmetry in the elastic scattering of longitudinally polarized electrons on unpolarized protons.
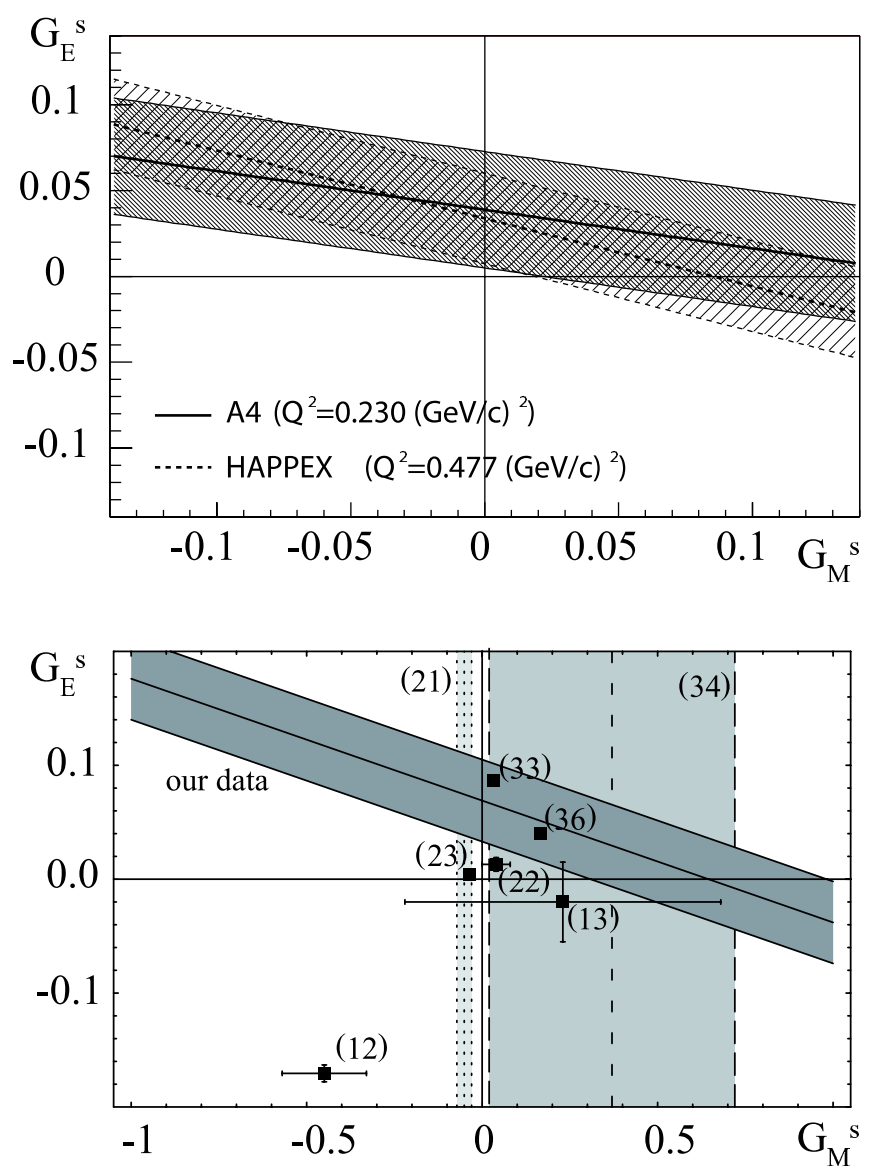

Fig. 8. Top: The solid line represents all possible combinations of $G_{E}^{s}+0.225 G_{M}^{s}$ as extracted from the work presented here at a $Q^{2}$ of $0.230(\mathrm{GeV} / c)^{2}$. The densely hatched region represents the 1- $\sigma$ uncertainty. The recalculated result from the HAPPEX published asymmetry at $Q^{2}$ of $0.477(\mathrm{GeV} / c)^{2}$ is indicated by the dashed line, the less densely hatched area represents the associated error of the HAPPEX result. Bottom: The solid lines represent the result on $G_{E}^{s}+0.106 G_{M}^{s}$ as extracted from our new data at $Q^{2}=0.108(\mathrm{GeV} / c)^{2}$ presented here. The hatched region represents in all cases the one- $\sigma$-uncertainty with statistical and systematic and theory error added in quadrature. The dashed lines represent the result on $G_{M}^{s}$ from the SAMPLE experiment [31]. The dotted lines represent the result of a recent lattice gauge theory calculation for $\mu_{s}[32]$. The boxes represent different model calculations and the numbers denote the references.

\section{Conclusion}

From the difference between the measured $A_{L R}(e p)$ and the theoretical prediction in the framework of the Standard Model, $A_{0}$, we extract a linear combination of the strange electric and magnetic form factors for the $570.4 \mathrm{MeV}$ data at a $Q^{2}$ of $0.108(\mathrm{GeV} / c)^{2}$ of $G_{E}^{s} \pm$ $0.106 G_{M}^{s}=0.071 \pm 0.036$. For the data at $854.3 \mathrm{MeV}$ corresponding to a $Q^{2}$ value of $0.230(\mathrm{GeV} / c)^{2}$ we extract $G_{E}^{s}+0.225 G_{M}^{s}=0.039 \pm 0.034$. Statistical and systematic error of the measured asymmetry and the error in the theoretical prediction of $A_{0}$ been added in quadrature. In fig. 8 the results for the $570.4 \mathrm{MeV}$ data are displayed. 


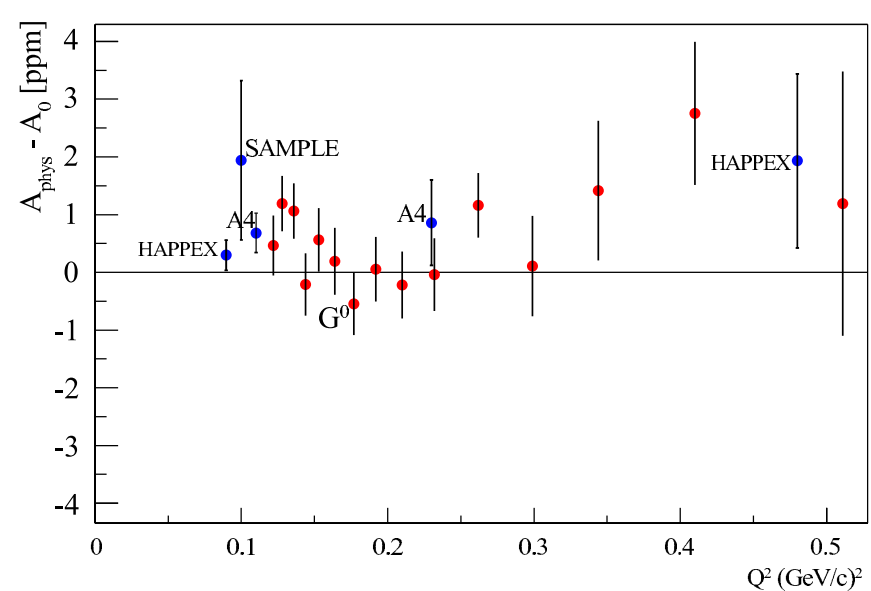

Fig. 9. A compilation of the world PV asymmetry data. The plot shows the difference $\left(A_{\text {phys }}-A_{0}\right)$ between the published measured PV physics asymmetries $A_{\text {phys }}$ and the asymmetry $A_{0}$ expected in the standard model assuming no strangeness. A significant difference is a direct sign for strangeness contribution. The blue points have been measured detecting the scattered electron, the red points denote the G0 experiment results where the proton has been detected under forward angles.

A recent very accurate determination of the strangeness contribution to the magnetic moment of the proton $\mu_{s}=$ $G_{M}^{s}\left(Q^{2}=0(\mathrm{GeV} / c)^{2}\right)$ from lattice gauge theory [32] would yield a larger value of $G_{E}^{s}=0.076 \pm 0.036$ if the $Q^{2}$ dependence from 0 to $0.108(\mathrm{GeV} / c)^{2}$ is neglected. The theoretical expectations for another quenched lattice gauge theory calculation [33], for $S U(3)$ chiral perturbation theory [34], from a chiral soliton model [35], from a quark model [36], from a Skyrme-type soliton model [37] and from an updated vector meson dominance model fit to the form factors [38] are included in fig. 8.

Recently the HAPPEX- and the G0-collaboration published measurements which are reviewed in a different contribution to these proceedings. Figure 9 shows a compilation of the world data in the $Q^{2}$-range up to $0.5(\mathrm{GeV} / c)^{2}$. The plot shows the difference $\left(A_{\text {phys }}-A_{0}\right)$ between the published measured PV physics asymmetries $A_{\text {phys }}$ and the asymmetry $A_{0}$ expected in the standard model assuming no strangeness. A significant difference is a direct sign for strangeness contribution. The blue points have been measured detecting the scattered electron, the red points denote the G0 experiment results where the proton has been detected under forward angles.

Combining the HAPPEX data at $Q^{2}=0.1(\mathrm{GeV} / c)^{2}$ and the extrapolated G0 data and with our previous data at this $Q^{2}$ value gives further constraints on $G_{E}^{s}$ and $G_{M}^{s}$. Figure 10 shows the present status of the world data at $Q^{2}=0.1(\mathrm{GeV} / c)^{2}$ in the $G_{E}^{s}$ versus $\mathrm{s} G_{M}^{s}$ plane. The SAMPLE data have been measured at backward angle. A4, HAPPEX and G0 at forward angle. The HAPPEX He data have been measured using a ${ }^{4}$ He target, which has no magnetic form factor due to the fact that the nucleus has spin 0 . One can extract a value for both $G_{E}^{s}$ and $G_{M}^{s}$ separately: $G_{M}^{s}=0.62 \pm 0.31$ and $G_{E}^{s}=-0.012 \pm 0.029$. While the combined value for $G_{E}^{s}$ has a sensitivity which

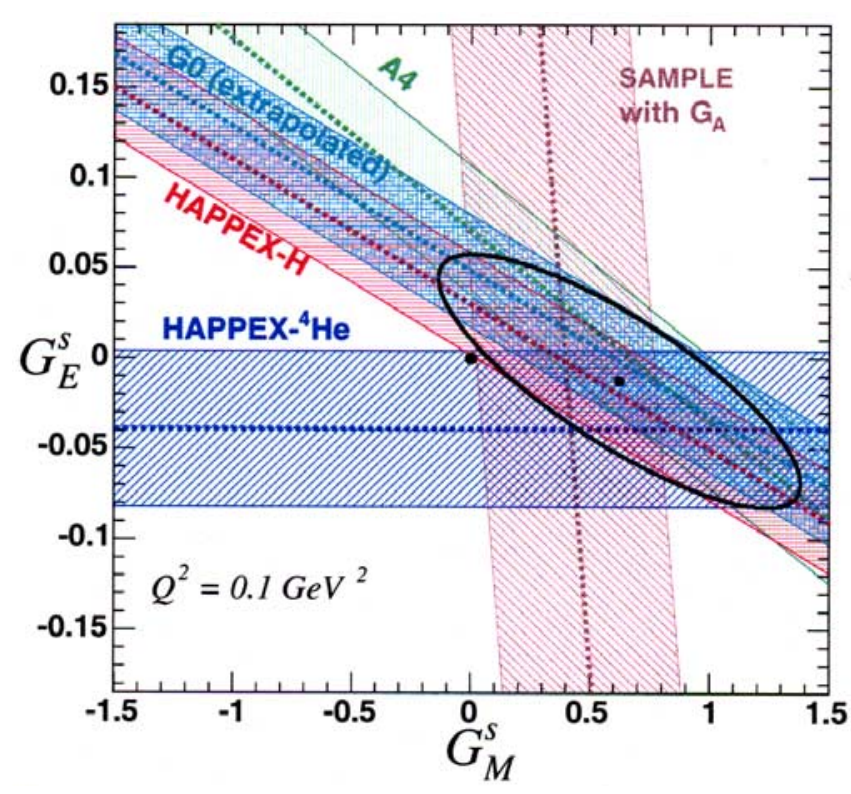

Fig. 10. The combination of all available data at forward and backward angles at from $\mathrm{H}$ and He target constrains the possible values for $G_{E}^{s}$ and $G_{M}^{s}$. The ellipse gives the two sigma contour plot.

is now reaching the accuracy of the electromagnetic form factors, the error on $G_{M}^{s}$ is still very large.

\section{Perspectives}

Further constrains on $G_{M}^{s}$ can be expected from further measurements combining $\mathrm{H}$ and $\mathrm{He}$ data at forward angles, as planned by the HAPPEX collaboration. A different approach is to combine $\mathrm{H}$ and $\mathrm{D}$ data at backward angles. We are preparing a series of measurements of the parity-violating asymmetry in the scattering of longitudinally polarized electrons off unpolarized protons and deuterons under backward scattering angles of $140^{\circ}<$ $\theta_{e}<150^{\circ}$ with the A4 apparatus at a $Q^{2}$ of $0.23(\mathrm{GeV} / c)^{2}$ in order to separate the electric $\left(G_{E}^{s}\right)$ and magnetic $\left(G_{M}^{s}\right)$ strangeness contribution to the electromagnetic form factors of the nucleon. We have changed the experimental setup. The detector has been put on a rotatable platform, construction work and reinstallation of detector and 2028 cables in the experimental hall had been finished by April 2005. We have found a large background stemming from photons coming from $\pi^{0}$-decay. The energy range of those photons cover partly the same energy interval as the energy range of the elastic scattered electrons. Our electromagnetic shower calorimeter has the same response function for electrons as for photons. $\pi^{0}$-production has its own unknown parity-violating asymmetry. In order to avoid pollution of our elastic signal with photons from $\pi^{0}$ decay, we have installed an electron tagging system of 72 additional plastic scintillators between scattering chamber and $\mathrm{PbF}_{2}$-crystals. Figure 11 shows a schematic of the 


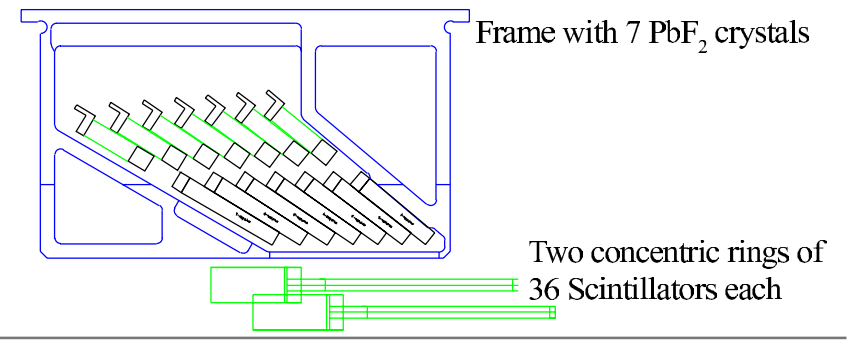

Vacuum of Scatering Chamber

Fig. 11. The drawing shows a cut through the upper part of the A4 detector system. In the upper part, one sees the aluminum frame with seven $\mathrm{PbF}_{2}$-crystals. The scintillator system for detecting electrons in coincidence with the calorimeter is located between the $\mathrm{PbF}_{2}$-crystals and the vacuum chamber. Two concentrical rings contain 36 scintillator each. The electron rates are low enmough, so that one scintillator can cover $14 \mathrm{PbF}_{2}$-crystals, i.e. two aluminum frames.

setup. The scintillators are arranged in two rings symmetrically around the scattering chamber. Only charged particle traversing the scintillator produce an output signal. If a scintillator produces a signal, it is converted to a logical level which then serves for the short coincidence time of $25 \mathrm{~ns}$ as an additional bit in the histogramming circuit of the readout electronics. If the bit is set, and in addition a signal arrives from the $\mathrm{PbF}_{2}$-calorimeter, the event is histogrammed into one region of the histogramming memory. If there is an event in the calorimeter without tagging bit from the scintillators, it is histogrammed into a different memory address range. Figure 12 shows 7 typical $\mathrm{PbF}_{\mathrm{s}}$ calorimeter spectra taken with the electron tagging scintillator system. The blue histograms show electron spectra where the additional scintillator system had given a coinci-

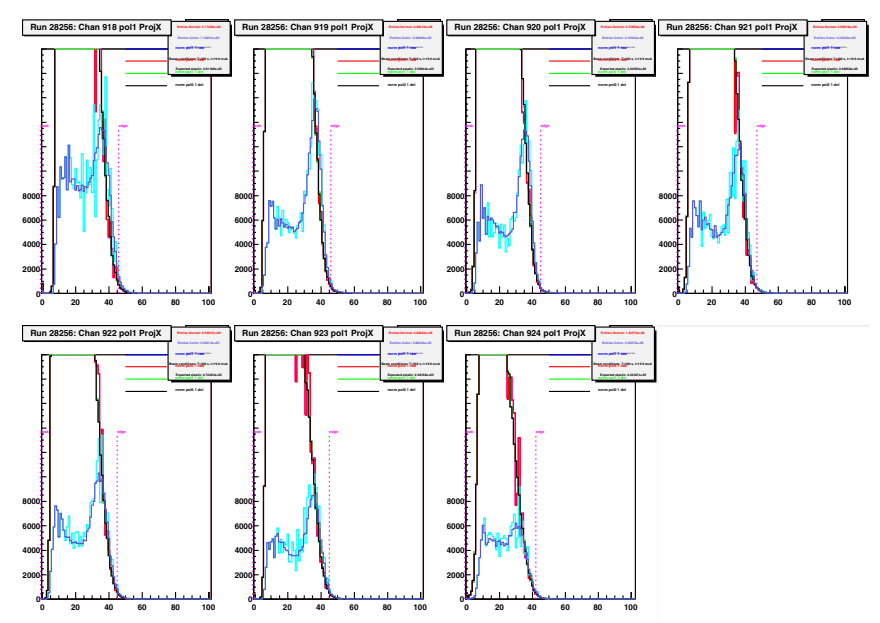

Fig. 12. The blue histograms show electron spectra where the additional scintillator system had given a coincidence signal. They exhibit a clear elastic peak with a good signal-tobackground ratio. The black and red histograms (only partly shown) correspond to the large background from photons from $\pi^{0}$-decay. Both spectra are taken always in parallel for better control of systematic effects. dence signal. They exhibit a clear elastic peak with a good signal-to-background ratio. The count rate under the elastic peak corresponds to $80 \%-90 \%$ of the expected elastic count rate. At forward angles, this ratio had been $70 \%$. We expect at backward angles a higher efficiency since the radiative tail is less pronounced at the beam energy of $315.13 \mathrm{MeV}$. The black and red histograms (only partly shown) correspond to the large background from photons from $\pi^{0}$-decay. For these signals the scintillator system had no coincident signal. The sum of both spectra would give the total spectrum. The system will be commissioned in December 2005. Data taking will start in January 2006. We plan to accumulate $1000 \mathrm{~h}$ with hydrogen target corresponding to an error in $G_{M}^{s}$ of \pm 0.13 , corresponding to a factor of three over the SAMPLE result and a factor of two improvement over the present combined world data at $Q^{2}=0.1(\mathrm{GeV} / c)^{2}$. In order to improve the understanding on the systematical uncertainty coming from the axial form factor, we will also collect the same amount of statistics with a deuterium target.

This work has been supported by the DFG in the framework of the SFB 201 and SPP 1034. We are indebted to K.H. Kaiser and the whole MAMI crew for their tireless effort to provide us with good electron beam. We are also indebted to the A1Collaboration for the use of the Møller polarimeter. My deep thanks got to H. Arenhövel, H. Backe, D. Drechsel, J. Friedrich, K.H. Kaiser and Th. Walcher. During the last ten years at the Institut für Kernphysik it had been a pleasure to work with them. I want to thank them not only for their continuous encouragement but also for many stimulating discussions and also for keeping a lifely, scientific spirit at the institute.

\section{References}

1. T.D. Lee, C.N. Yang, Phys. Rev. 104, 254 (1956).

2. C.S. Wu et al., Phys. Rev. 105, 1413 (1957).

3. T.R. Prescott et al., Phys. Lett. B 84, 524 (1979).

4. P.A. Souder et al., Phys. Rev. Lett. 65, 694 (1990).

5. J. Ahrens et al., Bucl. Phys. A 446, 377c (1985).

6. S. Kox, D. Lhuillier, F. Maas, J. Van de Wiele (Editors), From Parity Violation to Hadronic Structure and More, Proceedings of PAVI2004, Eur. Phys. J. A 24, s02 (2005).

7. T.R. Donoghue, E. Golowich, B.R. Holstein, Dynamics of the Standard Model, first paperback edition (with corrections) (Cambridge University Press, Cambridge, 1992).

8. H.D. Politzer, Phys. Rev. Lett. 30, 1346 (1973).

9. D.J. Gross, F. Wilczek, Phys. Rev. Lett. 30, 1343 (1973).

10. M. Jamin, Phys. Lett. B 538, 71 (2002).

11. M.M. Pavan et al., PiN Newslett. 16, 110 (2002).

12. X.-D. Ji, Phys. Rev. Lett. 74, 1071 (1995).

13. M. Goncharov et al., Phys. Rev. D 64, 112006 (2001).

14. M. Tzanov et al., Nutev neutrino dis., hep-ex/0306035, 2003.

15. M. Glück, E. Reya, A. Vogt. Eur. Phys. J. C 5, 461 (1998).

16. E. Leader et al., Phys. Rev. D 67, 074017 (2003).

17. D.B. Kaplan et al., Nucl. Phys. B 310, 527 (1988).

18. M.J. Musolf et al., Phys. Rep. 239, 1 (1994).

19. J. Friedrich, Th. Walcher, Eur. Phys. J. A 17, 607 (2003).

20. W.J. Marciano, A. Sirlin, Phys. Rev. D 29, 75 (1984). 
21. S. Eidelmann et al., Review of particle properties, Phys. Lett. B 592, 1 (2004).

22. S.-L. Zhu et al., Phys. Rev. D 62, 033008 (2000).

23. G. Küster, H. Arenhövel, Nucl. Phys. A 626, 911 (1997).

24. K. Aulenbacher et al., Nucl. Instrum. Methods A 391, 498 (1997).

25. M. Seidl et al., High precision beam energy stabilisation of the Mainz microtron MAMI, in Proceedings of the EPAC 2000 (2000) p. 1930.

26. P. Bartsch, Aufbau eines Møeller-Polarimeters für die Drei-Spektrometer-Anlage und Messung der Helizitätsasymmetrie in der Reaktion $p\left(\boldsymbol{e}, e^{\prime} p\right) \pi^{0}$ im Bereich der $\Delta$-Resonanz, Dissertation Mainz, 2001.

27. F.E. Maas et al., Proceedings of the ICATPP-7 (World Scientific, 2002) p. 758.
28. P. Achenbach et al., Nucl. Instrum. Methods A 465, 318 (2001).

29. F.E. Maas et al., Phys. Rev. Lett. 93, 022002 (2004).

30. F.E. Maas et al., Evidence for strange quark contributions to the nucleon's form-factors at $q^{2}=0.108(\mathrm{Gev} / \mathrm{c})^{2}$, nuclex/0412030, 2004.

31. D.T. Spayde et al., Phys. Lett. B 583, 79 (2004).

32. D.B. Leinweber et al., Phys. Rev. Lett. 94, 212001 (2005).

33. R. Lewis et al., Phys. Rev. D 67, 013003 (2003).

34. T.R. Hemmert et al., Phys. Rev. C 60, 045501 (1999).

35. A. Silva et al., Eur. Phys. J. A 22, 481 (2004).

36. V. Lyubovitskij et al., Phys. Rev. C 66, 055204 (2002).

37. H. Weigel et al., Phys. Lett. B 353, 20 (1995).

38. H.-W. Hammer et al., Phys. Rev. C 60, 045204 (1999). 